\title{
Association between lysyl oxidase and fibrotic focus in relation with inflammation in breast cancer
}

\author{
YOUNG JU JEONG ${ }^{1}$, SUNG HWAN PARK ${ }^{1}$, SUNG HEE MUN ${ }^{2}$, \\ SANG GYU KWAK ${ }^{3}$, SUN-JAE LEE ${ }^{4}$ and HOON KYU OH ${ }^{4}$ \\ Departments of ${ }^{1}$ Surgery, ${ }^{2}$ Radiology, ${ }^{3}$ Medical Statistics and ${ }^{4}$ Pathology, \\ College of Medicine, Catholic University of Daegu, Daegu 705-718, Repulic of South Korea
}

Received October 25, 2016; Accepted August 3, 2017

DOI: 10.3892/ol.2017.7617

\begin{abstract}
We hypothesized that lysyl oxidase (LOX) contributes to the formation of fibrotic focus (FF) in association with inflammation and serves a significant role in breast carcinogenesis. In the present study, the association between the expression of LOX family members and FF with regards to with inflammation was analyzed, and the prognostic significance of LOX and FF in breast cancer was investigated. Immunohistochemical staining for LOX, LOX-like protein (LOXL) 1, LOXL2 and LOXL3 was performed in primary breast cancer tissues. The status of FF within the tumor was assessed, including size and grade. Levels of inflammatory markers, intratumoral and peritumoral lymphocyte infiltration were also evaluated. The clinicopathological characteristics were evaluated from the medical records of patients. In the present study, the expression of LOX family members was not associated with the presence of FF. FF was identified to be associated with intratumoral and peritumoral inflammation, tumor stage, larger tumor size, lymph node metastasis, high histologic grade, and p53 expression. LOX and LOXL3 were associated with intratumoral, and peritumoral inflammation. Furthermore, LOXL1 was associated with intratumoral inflammation and interleukin-4. In addition, LOX was associated with cluster of differentiation $8^{+} \mathrm{T}$ cells. LOXL3 was associated with expression of ER and PR, and molecular subtype. In the survival analysis, overall survival time was statistically significantly longer in the FF-negative compared with that in the FF-positive group. In conclusion, it was demonstrated that FF and the expression of LOX family members were associated with inflammation in breast cancer. FF was associated with poor prognostic markers of breast cancer. Further studies are required to clarify the mechanisms underlying the
\end{abstract}

Correspondence to: Professor Hoon Kyu Oh, Department of Pathology, College of Medicine, Catholic University of Daegu, 3056-6 Daemyoung-4-dong, Nam-gu, Daegu 705-718, Republic of Korea

E-mail: ap510@cu.ac.kr

Key words: lysyl oxidase, LOX, fibrotic focus, inflammation, breast cancer association between the LOX family, FF and inflammation in breast cancer.

\section{Introduction}

Carcinogenesis is a complex process forming a cancer and is determined by cellular, genetic and epigenetic changes. In recent years, various factors have been investigated to reveal the mechanism of carcinogenesis and especially tumor microenvironment has been recognized as an important factor in carcinogenesis. One of the main components of tumor microenvironment is the extracellular matrix (ECM) remodeling $(1,2)$. The ECM is defined as a complex structure build by interacting extracellular molecules including proteoglycans, polysaccharides, fibronectin, laminin and fibers such as collagen and elastin. The ECM provides structural and mechanical support to cells and tissues (1). During carcinogenesis, the composition and the overall content of the ECM change and the ECM is progressively stiffened $(3,4)$. ECM stiffness has been implicated to promote tumor progression (5) and is partially associated with lysyl oxidase (LOX)-mediated collagen cross-linking $(2,5)$.

LOX is a copper-containing amine oxidase that catalyzes lysine-derived cross-links in collagen and elastin and stabilizes the ECM (6). For many years, LOX has been demonstrated that it has diverse functions including the ability regulating gene transcription, cell growth control, cell motility and migration and cell adhesions (7). LOX is encoded by the LOX gene located on chromosome 5 (5q23.3-31.2) and belongs to a copper dependent amine-oxidase family currently consisting of five members (LOX, LOX-like protein (LOXL) 1, LOXL2, LOXL3 and LOXL4) (6,8-11). Recent studies have shown that LOX family is related to tumor fibrosis, invasion and metastasis (10-13).

ECM stiffness is caused by collagen deposition and linearization and bundling of interstitial collagen (4) and LOX has a crucial role in stiffness-associated tumor progression $(5,10,14)$. ECM remodeling including stiffness contributes to fibrotic changes in tumor (15), which is defined as fibrotic focus (FF). Since Hasebe et al (16) have proposed FF as an indicator of tumor aggressiveness in invasive breast cancer, many studies have shown the relationship between FF and the prognosis of breast cancer (17). 
Inflammation has been also recognized as an important component of tumorigenesis, and it usually precedes fibrosis (18). Recently, it has been described that inflammation plays an important role in fibrosis as an inducer of epithelial-mesenchymal transition (EMT) in cancer (19). Also, it has been suggested that invasion and aggression of breast cancer correlates with ECM stiffening and immune cell infiltration (4).

Nevertheless, the mechanisms of LOX-mediated tumor progression in association with fibrosis and inflammation are not fully understood. In this study, we analyzed the association between the expression of the members of the LOX family and FF in relation with inflammation, and investigated the prognostic significance of the members of the LOX family and FF in human breast cancer.

\section{Materials and methods}

Patients and materials. Patients with primary breast cancer who underwent surgery between January 2003 and December 2010 at the Daegu Catholic University Hospital (Daegu, Korea) were enrolled. Formalin-fixed and paraffin-embedded specimens from the patients were stained with hematoxylin and eosin (H\&E) and histologically examined and reviewed by an experienced pathologist. The clinicopathologic characteristics were evaluated based on the pathologic reports and the medical records. Disease stage was assessed according to the seventh edition of the American Joint Committee on Cancer (AJCC) staging manual for breast cancer. Molecular subtype of the breast cancer was classified according to the immunohistochemical findings for estrogen receptor (ER), progesterone receptor (PR), human epidermal growth factor receptor 2 (HER2) and Ki-67 labeling index. The informed written consent was obtained from all patients for the use of their data. The ethics review of the study was waived from the Institutional Review Board at the Daegu Catholic University Hospital according to the deliberation criteria.

Tissue microarray and immunohistochemistry. Representative paraffin blocks of invasive breast carcinomas were selected to prepare for tissue microarray (TMA) and TMA was constructed following the methods described in our previous study (20). TMA blocks were cut into $5 \mu \mathrm{m}$-thick sections and immunohistochemical staining was performed on the TMA sections using the Bond Polymer Intense Detection System (Leica Microsystems, Victoria, Australia) according to the manufacturer's instruction with minor modifications.

Immunohistochemical staining for the members of the LOX family were conducted using commercially available primary antibodies, LOX (1:200, ab31238; Abcam, Cambridge, MA, USA), LOXL1 (1:100, NBP1-82827; Novus Biologicals, Littleton, CO, USA), LOXL2 (1:100, NBP1-32954; Novus Biologicals) and LOXL3 (1:100, NBP1-85908; Novus Biologicals). The TMA sections were also immunostained for CD4 (RTU, clone 4B12; Leica Biosystems, Wetzlar, Germany), CD8 (1:200, clone C8/144B; Dako, Glostrup, Denmark), CD68 (1;200, clone PG-M1; Dako), ER (1:100, clone 6F11; Novocastra, Newcastle, UK), PR (1:100, clone 16; Novocastra), HER 2 (1:250, A0485; Dako), Ki-67 (1:200, MM1-L; Novocastra), Bcl-2 (1:4, clone 124; Dako), p53 (1:200, BP53.12;
Zymed, Carlsbad, CA, USA) and epidermal growth factor receptor (EGFR) (1:100, clone EGFR.25; Novocastra). The levels of LOX, LOXL1, LOXL2 and LOXL3 assessed semiquantitatively as: 0 (no staining); 1+ (minimal intensity, $<10 \%$ of cells); $2+$ (moderate, $10-49 \%$ ); and $3+$ (marked, $\geq 50 \%)$. The expression level 0 and 1 were designated as negative and 2 and 3 as positive. Fig. 1 shows representative microphotographs of immunohistochemical expression of LOX, LOXL1, LOXL2 and LOXL3. The number of cluster of differentiation (CD) $4^{+}$ $\mathrm{T}$ cells, $\mathrm{CD}^{+} \mathrm{T}$ cells and $\mathrm{CD}^{+} 8^{+}$macrophages was evaluated under a microscopic and counted both in the stroma and the cancer cell nest. Intratumoral (within the tumor boundary) and peritumoral (at the edge of tumor boundary) lymphocyte infiltration was also assessed semiquantitatively as follows: 0 (no or scant lymphocytes); 1 (a few scattered lymphocytic infiltration); 2 (scattered lymphocytic aggregation); and 3 (diffuse and dense aggregation of lymphocytes), where 1, 2 and 3 are designated as positive and 0 is as negative.

FF was diagnosed when there was a scar-like area or radially expanding fibrous bands consist of the fibroblasts and collagen fibers within the tumor, and surrounded by a highly cellular zone of infiltrating carcinoma cells (17). Fig. 2 shows representative example of $\mathrm{FF}$ in $\mathrm{H} \& \mathrm{E}$ stained breast cancer specimen. The status of FF within the tumor was assessed including size and grade and a minimal dimension of $1 \mathrm{~mm}$ of a fibrosclerotic core was referred to as FF.

$R T-P C R$. The levels of cytokines including tumor necrosis factor (TNF)- $\alpha$, interleukin (IL)-1, IL-2, IL-4, IL-6, transforming growth factor (TGF)- $\beta$, interferon (IFN)- $\gamma$ and nuclear factor (NF) $-\kappa \mathrm{B}$ p50 were assessed by the levels of RNA transcripts in frozen tissue using RT-PCR. Total RNA was isolated from frozen breast cancer tissues using TRIzol reagent (Invitrogen; Thermo Fisher Scientific, Inc., Waltham, MA, USA) according to the manufacturer's protocol. Reverse transcription of total RNA was performed using a commercial kit (Superscript II RNase H-reverse transcriptase; Invitrogen; Thermo Fisher Scientific, Inc.). PCR amplification was performed with specific primers and the sequences of primers are presented in Table I. PCR products were analyzed by agarose gel electrophoresis and visualized by ethidium bromide staining.

Statistical analysis. Statistical analyses were performed using SPSS software version 19.0 (SPSS, Inc., Chicago, IL, USA). Association between FF and LOX, LOXL1, LOXL2 and LOXL3 was analyzed using the Chi-square test. Association of FF with number of inflammatory cells was assessed using two sample t-test. Association of FF with the clinicopathologic characteristics was analyzed using two sample t-test and the Chi-square test. Binary logistic regression analysis was performed to assess the odds ratios (ORs) of the statistically significant factors in univariate analysis. Association of the members of the LOX family with number of inflammatory cells was assessed using two sample t-test. Association between the members of the LOX family with the clinicopathologic characteristics was analyzed using two sample t-test and the Chi-square test. Survival data were analyzed using Kaplan-Meier survival analysis. $\mathrm{P}<0.05$ was considered to indicate a statistically significant difference. 
Table I. Sequences of primers used in PCR.

\begin{tabular}{llc}
\hline Primer name & \multicolumn{1}{c}{ Primer sequence $\left(5^{\prime} \rightarrow 3^{\prime}\right)$} & Orientation \\
\hline TNF- $\alpha$ & CCCTCAACCTCTTCTGGCTC & Forward \\
TNF- $\alpha$ & AGGCAGCTCCTACATTGGGT & Reverse \\
IL-1 & AAATACCTGTGGCCTTGGGC & Forward \\
IL-1 & TTTGGGATCTACACTCTCCAGCT & Reverse \\
IL- 2 & GCAACTCCTGTCTTGCATTG & Forward \\
IL- 2 & TGCTTTGACAAAAGGTAATCCA & Reverse \\
IL-4 & ATGGGTCTCACCTCCCAACTGC & Forward \\
IL-4 & TTCCTGTCGAGCCGTTTCAG & Reverse \\
IL-6 & TACCCCCAGGAGAAGATTCC & Forward \\
IL-6 & AAAGCTGCGCAGAATGAGAT & Reverse \\
TGF- $\beta$ & CCCAGCATCTGCAAAGCTC & Forward \\
TGF- $\beta$ & GTCAATGTACAGCTGCCGCA & Reverse \\
IFN- $\gamma$ & AGTTATATCTTGGCTTTTCA & Forward \\
IFN- $\gamma$ & ACCGAATAATTAGTCAGCTT & Reverse \\
NF- $-\kappa$ p p 50 & CACCTAGCTGCCAAAGAAGG & Forward \\
\hline
\end{tabular}

TNF, tumor necrosis factor; IL, interleukin; TGF, transforming growth factor; IFN, interferon; NF- $\kappa \mathrm{B}$, nuclear factor- $\mathrm{kB}$

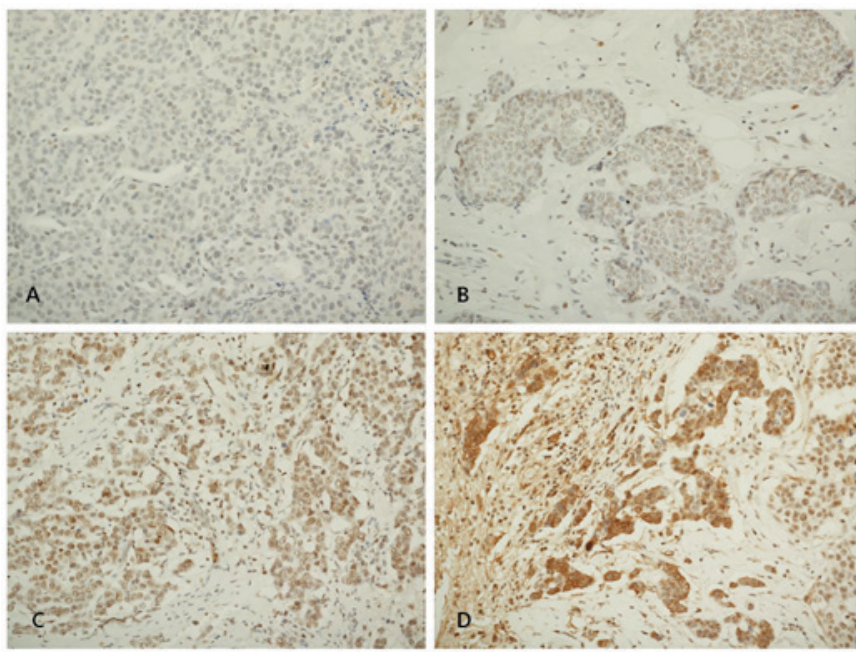

Figure 1. Representative immunohistochemical expression of the members of the lysyl oxidase (LOX) family in invasive breast carcinoma. (A) Negative expression of LOX protein, (B) minimal expression of LOX-like protein (LOXL) 1 protein, (C) moderate expression of LOXL2 protein, (D) marked expression of LOXL3 protein (immunohistochemical staining; magnification, $\mathrm{x} 200)$.

\section{Results}

Clinicopathologic characteristics of the patients. A total of 291 patients with invasive breast cancer were included in this study. The mean age of the patients was $52.63 \pm 11.01$ years (range, 24-90 years). Clinicopathologic characteristics of the patients are shown in Table II. Among the patients, the most common histologic type of invasive cancer was invasive ductal carcinoma not otherwise specified $(89.7 \%)$, and the remaining were infiltrating lobular carcinoma $(n=7)$, papillary carcinoma

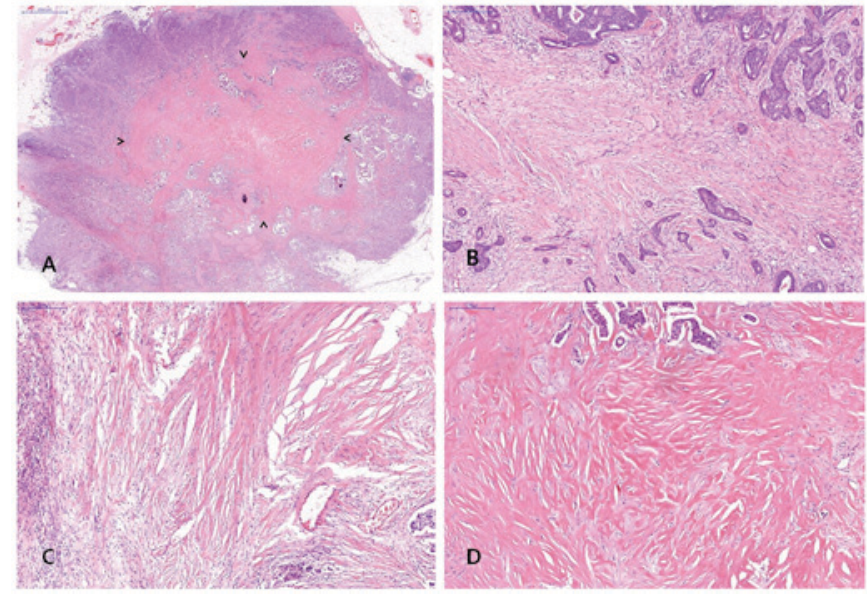

Figure 2. Representative histology of fibrotic focus (FF) in invasive breast carcinoma. (A) Representative histology of FF; arrow heads indicate area of FF in invasive breast carcinoma [hematoxylin and eosin (H\&E); magnification, x10], (B) FF with fibrosis grade 1 showing fibroblastic proliferation in stroma with small amount of collagen (H\&E; magnification, x100), (C) FF with fibrosis grade 2 which intermediate between grade 1 and 3 fibrosis (H\&E; magnification, x100), (D) FF with fibrosis grade 3 showing mostly hyalinized collagenous stroma (H\&E; magnification, $\mathrm{x} 100)$.

$(n=4)$, micropapillary carcinoma $(n=2)$, mucinous carcinoma $(n=4)$, tubular carcinoma $(n=4)$, metaplastic carcinoma $(n=3)$, medullary carcinoma $(n=2)$, sarcoma $(n=1)$, inflammatory carcinoma $(n=1)$ and mixed type $(n=2)$.

Association between FF and LOX families. The percentage of positive FF was $37.5 \%$ and positive rate of LOX expression was $49.1 \%$ in primary breast cancer tissues. The expression of LOX, LOXL1, LOXL2 and LOXL3 was not associated with presence of FF (Table III). 
Table II. Patient characteristics.

Clinicopathologic variables
Age (years), mean (range)
Menopausal status, n (\%)
Premenopausal
Postmenopausal
Tumor size (cm), mean (range)
Histologic grade, n (\%)
I
II
III

Lymph node metastasis, n (\%)

Negative

Positive

$165(62.26)$

100 (37.74)

Stage, n $(\%)$

I

II

III

IV

$51.81 \pm 11.29(24-90)$
$143(49.14)$
$148(50.86)$
$2.09 \pm 1.80(0.1-23.0)$
$64(22.15)$
$110(38.06)$
$115(39.79)$

$126(43.9)$

117 (40.77)

$36(12.54)$

8 (2.79)

Lymphovascular invasion, n (\%)

Negative

Positive

197 (68.4)

91 (31.6)

ER, n (\%)

Negative

Positive

$102(35.17)$

188 (64.83)

PR, n (\%)

Negative

Positive

$81(27.93)$

209 (72.07)

HER2 overexpression, n (\%)

Negative

Positive

$\mathrm{Ki}-67, \mathrm{n}(\%)$

$<14 \%$

$\geq 14 \%$

$117(50.87)$

114 (49.13)

129 (58.64)

$91(41.36)$

Molecular subtype, n (\%)

Luminal A

Luminal B

HER 2

Basal-like

$113(39.65)$

$110(38.6)$

$26(9.12)$

$36(12.63)$

Fibrotic focus, n (\%)

Negative

Positive

LOX, n (\%)

Negative

Positive

LOXL1, n (\%)

Negative

Positive

$200(68.73)$

$91(31.27)$

LOXL2, n (\%)

Negative

$176(60.48)$

Positive

$178(62.46)$

107 (37.54)

$148(50.86)$

143 (49.14)

115 (39.52)
Table II. Continued.

\begin{tabular}{lr}
\hline Clinicopathologic variables & \multicolumn{1}{l}{ Value } \\
\hline LOXL3, n (\%) & $252(86.6)$ \\
Negative & $39(13.4)$ \\
Positive & \\
Intratumoral inflammation, n (\%) & $51(17.53)$ \\
Negative & $240(82.47)$ \\
Positive & \\
Peritumoral inflammation, n (\%) & $31(10.65)$ \\
Negative & $260(89.35)$ \\
Positive & \\
\hline
\end{tabular}

ER, estrogen receptor; PR, progesterone receptor; HER2, human epidermal growth factor receptor 2; LOX, lysyl oxidase; LOXL, LOX-like protein.

Table III. Association between the fibrotic focus and LOX families.

\begin{tabular}{llll}
\hline & \multicolumn{2}{c}{ Fibrotic focus } & \\
\cline { 2 - 3 } Variables & Negative & Positive & P-value \\
\hline LOX, n (\%) & & & \\
Negative & $92(51.69)$ & $52(48.6)$ & 0.614 \\
Positive & $86(48.31)$ & $55(51.4)$ & \\
LOXL1, n (\%) & & & \\
Negative & $124(69.66)$ & $71(66.36)$ & 0.561 \\
Positive & $54(30.34)$ & $36(33.64)$ & \\
LOXL2, n (\%) & & & \\
$\quad$ Negative & $112(62.92)$ & $59(55.14)$ & 0.194 \\
Positive & $66(37.08)$ & $48(44.86)$ & \\
LOXL3, n (\%) & & & \\
$\quad$ Negative & $153(85.96)$ & $94(87.85)$ & 0.649 \\
Positive & $25(14.04)$ & $13(12.15)$ & \\
\hline
\end{tabular}

LOX, lysyl oxidase; LOXL, LOX-like protein.
Association of FF with clinicopathologic features. By univariate analysis, FF was statistically significantly associated with stage II tumors $(\mathrm{P}<0.001)$, larger tumor size $(\mathrm{P}<0.001)$, lymph node metastasis $(\mathrm{P}<0.001)$, high histologic grade $(\mathrm{P}=0.001), \mathrm{p} 53 \quad(\mathrm{P}=0.018)$, intratumoral and peritumoral inflammation $(\mathrm{P}<0.001$ and $\mathrm{P}<0.001$, respectively) (Table IV). Multivariate analysis using covariate with $\mathrm{P}<0.2$ in univariate analysis was performed to assess the independent association of FF with clinicopathologic features. We found a significant association between FF and tumor size, histologic grade, lymph node metastasis and p53 (Table V).

Association of LOX families with clinicopathologic features. By univariate analysis, LOX was statistically significantly associated with intratumoral and peritumoral inflammation $(\mathrm{P}<0.001$ and $\mathrm{P}<0.001$, respectively) (Table VI). LOXL1 was 
Table IV. Association of fibrotic focus with clinicopathologic characteristics.

\begin{tabular}{|c|c|c|c|}
\hline \multirow[b]{2}{*}{ Clinicopathologic variables } & \multicolumn{2}{|c|}{ Fibrotic focus } & \multirow[b]{2}{*}{ P-value } \\
\hline & Negative & Positive & \\
\hline \multicolumn{4}{|l|}{ Tumor size, $\mathrm{n}(\%)$} \\
\hline$<2 \mathrm{~cm}$ & $120(67.42)$ & $38(35.51)$ & \multirow[t]{2}{*}{$<0.001^{\mathrm{a}}$} \\
\hline$\geq 2 \mathrm{~cm}$ & $58(32.58)$ & $69(65.49)$ & \\
\hline \multicolumn{4}{|l|}{ Histologic grade, $\mathrm{n}(\%)$} \\
\hline I & $52(29.38)$ & $10(9.34)$ & \multirow[t]{3}{*}{$<0.001^{\mathrm{a}}$} \\
\hline II & $64(36.16)$ & $45(42.06)$ & \\
\hline III & $61(34.46)$ & $52(48.60)$ & \\
\hline \multicolumn{4}{|l|}{ Lymph node metastasis, n (\%) } \\
\hline Negative & $126(71.19)$ & $43(40.19)$ & \multirow[t]{2}{*}{$<0.001$} \\
\hline Positive & $51(28.81)$ & $64(59.81)$ & \\
\hline \multicolumn{4}{|l|}{ Stage, n (\%) } \\
\hline I & $95(53.67)$ & $28(26.67)$ & \multirow[t]{4}{*}{$<0.001^{\mathrm{a}}$} \\
\hline II & $61(34.46)$ & $55(52.38)$ & \\
\hline III & $16(9.04)$ & $20(19.05)$ & \\
\hline IV & $5(2.83)$ & $2(1.90)$ & \\
\hline \multicolumn{4}{|c|}{ Lymphovascular invasion, $\mathrm{n}(\%)$} \\
\hline Negative & $136(76.84)$ & $57(53.27)$ & \multirow[t]{2}{*}{$<0.001$} \\
\hline Positive & $41(23.16)$ & $50(46.73)$ & \\
\hline \multicolumn{4}{|l|}{$\mathrm{ER}, \mathrm{n}(\%)$} \\
\hline Negative & $60(33.90)$ & $39(36.45)$ & \multirow[t]{2}{*}{0.662} \\
\hline Positive & $117(66.10)$ & $68(63.55)$ & \\
\hline \multicolumn{4}{|l|}{$\mathrm{PR}, \mathrm{n}(\%)$} \\
\hline Negative & $47(26.55)$ & $31(28.97)$ & \multirow[t]{2}{*}{0.658} \\
\hline Positive & $130(73.45)$ & $76(71.03)$ & \\
\hline \multicolumn{4}{|l|}{ HER2 overexpression, $\mathrm{n}(\%)$} \\
\hline Negative & $67(46.21)$ & $46(57.50)$ & \multirow[t]{2}{*}{0.105} \\
\hline Positive & $78(53.79)$ & $34(42.50)$ & \\
\hline \multicolumn{4}{|l|}{$\mathrm{Ki}-67, \mathrm{n}(\%)$} \\
\hline$<14 \%$ & $126(70.79)$ & $70(65.42)$ & \multirow[t]{2}{*}{0.344} \\
\hline$\geq 14 \%$ & $52(29.21)$ & $37(34.58)$ & \\
\hline \multicolumn{4}{|l|}{$\mathrm{p} 53, \mathrm{n}(\%)$} \\
\hline Negative & $49(27.53)$ & $44(41.12)$ & \multirow[t]{2}{*}{$0.018^{\mathrm{a}}$} \\
\hline Positive & $129(72.47)$ & $63(58.88)$ & \\
\hline \multicolumn{4}{|l|}{ Molecular subtype, n (\%) } \\
\hline Luminal A & $68(38.64)$ & $43(41.75)$ & 0.830 \\
\hline Luminal B & $71(40.34)$ & $38(36.89)$ & \\
\hline HER2 & $17(9.66)$ & $8(7.77)$ & \\
\hline Basal-like & $20(11.36)$ & $14(13.59)$ & \\
\hline Intratumoral inflammation, $\mathrm{n}$ & & & \\
\hline Negative & $39(21.91)$ & $7(6.54)$ & $0.001^{\mathrm{a}}$ \\
\hline Positive & $139(78.09)$ & $100(93.46)$ & \\
\hline Peritumoral inflammation, $\mathrm{n}$ & & & \\
\hline Negative & $24(13.48)$ & $2(1.87)$ & $0.001^{\mathrm{a}}$ \\
\hline Positive & $154(86.52)$ & $105(98.13)$ & \\
\hline
\end{tabular}

${ }^{a}$ Denotes statistical significance $(\mathrm{P}<0.05)$. ER, estrogen receptor; PR, progesterone receptor; HER2, human epidermal growth factor receptor 2 . 
Table V. Multivariate analysis of association between fibrotic focus and clinicopathologic characteristics.

\begin{tabular}{|c|c|c|c|}
\hline $\begin{array}{l}\text { Clinicopathologic } \\
\text { variables }\end{array}$ & OR & $95 \% \mathrm{CI}$ for OR & P-value \\
\hline
\end{tabular}

Tumor size, $\mathrm{n}(\%)$

$\begin{array}{cccc}<2 \mathrm{~cm} & 1 & - & - \\ \geq 2 \mathrm{~cm} & 4.409 & 1.726,11.264 & 0.002^{\mathrm{a}}\end{array}$

Histologic grade, $\mathrm{n}(\%)$

$$
\text { I }
$$

II

III

$\begin{array}{ccc}1 & - & - \\ 4.465 & 1.246,16.002 & 0.022^{\mathrm{a}} \\ 5.072 & 1.147,22.422 & 0.032^{\mathrm{a}}\end{array}$

Lymph node

metastasis, n (\%)

Negative

Positive

1
4.862

$1.727,13.687$

$0.003^{\mathrm{a}}$

Stage, n $(\%)$$$
\text { I }
$$

II

III

IV

1
.416
.306
.129

$\begin{array}{ll}0.115,1.502 & 0.180 \\ 0.057,1.636 & 0.166 \\ 0.012,1.395 & 0.092\end{array}$

HER2

overexpression, $\mathrm{n}(\%)$

\begin{tabular}{|c|c|c|c|}
\hline Negative & 1 & - & - \\
\hline Positive & .556 & $0.27,1.147$ & 0.112 \\
\hline \multicolumn{4}{|l|}{$\mathrm{p} 53, \mathrm{n}(\%)$} \\
\hline Negative & 1 & - & - \\
\hline Positive & .456 & $0.225,0.926$ & $0.030^{\mathrm{a}}$ \\
\hline \multicolumn{4}{|c|}{$\begin{array}{l}\text { Intratumoral } \\
\text { inflammation, n (\%) }\end{array}$} \\
\hline Negative & 1 & - & - \\
\hline Positive & 2.468 & $0.829,7.343$ & 0.104 \\
\hline \multicolumn{4}{|c|}{$\begin{array}{l}\text { Peritumoral } \\
\text { inflammation, n (\%) }\end{array}$} \\
\hline Negative & 1 & - & - \\
\hline Positive & 1.356 & $0.353,5.215$ & 0.658 \\
\hline
\end{tabular}

aDenotes statistical significance $(\mathrm{P}<0.05)$. HER2, human epidermal growth factor receptor 2; OR, odds ratio; CI, confidence interval.

statistically significantly associated with intratumoral inflammation $(\mathrm{P}=0.021)$. LOXL2 was also statistically significantly associated with intratumoral and peritumoral inflammation $(\mathrm{P}=0.004$ and $\mathrm{P}=0.041$, respectively). LOXL3 was significantly associated with positive expression of ER and PR $(\mathrm{P}<0.001$ and $\mathrm{P}<0.001$, respectively) and molecular subtype $(\mathrm{P}<0.001)$.

Association of FF and LOX families with inflammatory markers. Immunohistochemical staining for $\mathrm{CD}^{+} \mathrm{T}$ cells, $\mathrm{CD}^{+} \mathrm{T}$ cells and $\mathrm{CD}^{+} 8^{+}$macrophages were performed in 77 patients who had undergone surgery from January 2008 to December 2010. The levels of RNA transcripts for TNF- $\alpha$, IL-4 and NF- $\mathrm{B}$ p50 were analyzed in frozen tumor tissues although the expression levels of RNA transcripts for IL-1, IL-2, IL-6, TGF- $\beta$ and IFN- $\gamma$ were too low to be analyzed. In subgroup analysis, LOX and $\mathrm{CD}^{+} \mathrm{T}$ cell showed significant correlation $(\mathrm{P}=0.030)$ and LOXL1 and IL-4 showed significant association $(\mathrm{P}=0.019)$ (Table VII).

Association of FF and LOX family with patient outcomes. The median follow-up period was 72 months (range 1-147 months). Among 291 patients, 35 patients (12.0\%) showed tumor recurrence and 26 patients died from breast cancer. The 5-year survival rate was $90.0 \%$. Overall survival (OS) was statistically significantly longer in negative FF group than in positive FF group ( $\mathrm{P}=0.026)$ (Fig. 3A). Disease free survival (DFS) was longer in negative FF group although there was no statistical significance $(\mathrm{P}=0.054)$ (Fig. 3B). There was no significant association between LOX family and patients outcomes.

\section{Discussion}

Tumor fibrosis and inflammation have been increasingly recognized as important factors which influence tumor progression and metastasis. Recent studies have demonstrated that LOX is involved in creating fibrotic microenvironment and fibrosis-enhanced cancer metastasis $(5,21,22)$. Nevertheless, the relationship between LOX, FF, inflammation and tumor progression in human breast cancer have yet to be fully elucidated. FF is distinguished from organ fibrosis by the characteristics such as irregular or storiform patterned arrangement of fibroblast and collagen fibers, which shows radially expanding fibrous bands surrounded by a highly cellular zone of infiltrating carcinoma cells, and the location in the center of the tumor (17). In this study, we aimed to investigate whether LOX contribute to the formation of FF, especially in association with inflammation. There was no direct correlation between the members of the LOX family and FF in this study. However, FF, LOX, LOXL1 and LOXL2 showed significant correlation with intratumoral inflammation and FF, LOX and LOXL2 also related to peritumoral inflammation. These results suggest that the members of the LOX family and FF interact in relation with inflammation in breast cancer. Although the mechanisms by which LOX mediates FF formation in tumor are not clarified, functions of the LOX family in association with fibrosis in the cardiovascular system are relatively clear (23). LOX has been shown to be up-regulated by cytokines including TGF- $\beta$, TNF- $\alpha$ and IL-6 (23). Also, it has been implicated that LOX-mediated collagen cross-linking is critical in ECM stiffness (2,5). Acerbi et al (4) have shown that both ECM stiffness and cellular TGF- $\beta$ signaling correlated positively with immune cell infiltration in human breast cancer. Leight et al. have demonstrated that increased matrix stiffness regulates TGF- $\beta$ signaling, a potent inducer of EMT in tumor cells (24). In recent years, it has been suggested that EMT contributes to increase the collagen producing fibroblasts (21) and fibroblasts form FF in fibrotic tissues (17). Taken together these results also support our hypothesis. As far as I know, this is the first study to investigate the relationship between the members of the LOX family and FF.

It is well documented that organ fibrosis is associated with chronic inflammatory diseases $(18,19)$. A number of studies have suggested the role of inflammatory cells such as macrophage 
Table VI. Association of LOX families with clinicopathologic characteristics.

\begin{tabular}{|c|c|c|c|c|c|c|c|c|}
\hline \multirow[b]{2}{*}{$\begin{array}{l}\text { Clinicopathologic } \\
\text { variables }\end{array}$} & \multicolumn{2}{|c|}{ LOX } & \multicolumn{2}{|c|}{ LOXL1 } & \multicolumn{2}{|c|}{ LOXL2 } & \multicolumn{2}{|c|}{ LOXL3 } \\
\hline & $\begin{array}{c}\text { Positive } \\
\text { expression, } \\
\mathrm{n}(\%)\end{array}$ & P-value & $\begin{array}{c}\text { Positive } \\
\text { expression, } \\
\mathrm{n}(\%)\end{array}$ & P-value & $\begin{array}{c}\text { Positive } \\
\text { expression, } \\
\mathrm{n}(\%)\end{array}$ & $\mathrm{P}$-value & $\begin{array}{c}\text { Positive } \\
\text { expression, } \\
\mathrm{n}(\%)\end{array}$ & P-value \\
\hline Tumor size, n (\%) & & 0.496 & & 0.674 & & 0.740 & & 0.236 \\
\hline$<2 \mathrm{~cm}$ & $82(50.9)$ & & $52(32.3)$ & & $65(40.4)$ & & $25(15.5)$ & \\
\hline$\geq 2 \mathrm{~cm}$ & $61(46.9)$ & & $39(30.0)$ & & $50(38.5)$ & & $14(10.8)$ & \\
\hline Histologic grade, $\mathrm{n}(\%)$ & & 0.702 & & 0.549 & & 0.558 & & 0.263 \\
\hline I & $30(46.9)$ & & $18(27.7)$ & & $22(34.4)$ & & $5(7.8)$ & \\
\hline II & $52(47.3)$ & & $32(29.1)$ & & $44(40.0)$ & & $15(13.6)$ & \\
\hline III & $60(52.2)$ & & $40(34.8)$ & & $49(42.6)$ & & $19(16.5)$ & \\
\hline LN metastasis, $\mathrm{n}(\%)$ & & 0.596 & & 0.826 & & 0.367 & & 0.805 \\
\hline Negative & $82(47.7)$ & & $54(31.4)$ & & $65(37.8)$ & & $22(12.8)$ & \\
\hline Positive & $59(50.9)$ & & $35(30.2)$ & & $50(43.1)$ & & $16(13.8)$ & \\
\hline Stage, n (\%) & & 0.916 & & 0.930 & & 0.469 & & 0.752 \\
\hline I & $65(51.6)$ & & $41(32.5)$ & & $50(39.7)$ & & $16(12.7)$ & \\
\hline II & $55(47.0)$ & & 37 (31.6) & & $47(40.2)$ & & $15(12.8)$ & \\
\hline III & $18(50.0)$ & & $10(27.8)$ & & $15(41.7)$ & & 7 (19.4) & \\
\hline IV & $4(50.0)$ & & $2(25.0)$ & & $1(12.5)$ & & $1(12.5)$ & \\
\hline LVI, n (\%) & & 0.636 & & 0.505 & & 0.262 & & 0.905 \\
\hline Negative & $99(50.3)$ & & $64(32.5)$ & & $83(42.1)$ & & $27(13.7)$ & \\
\hline Positive & $43(47.3)$ & & $26(28.6)$ & & $32(35.2)$ & & $12(13.2)$ & \\
\hline $\mathrm{ER}, \mathrm{n}(\%)$ & & 0.469 & & 0.374 & & 0.465 & & $0.001^{\mathrm{a}}$ \\
\hline Negative & $47(46.1)$ & & $35(34.7)$ & & $43(42.2)$ & & $23(22.5)$ & \\
\hline Positive & $95(50.5)$ & & $55(29.3)$ & & $71(37.8)$ & & $16(8.5)$ & \\
\hline PR, n (\%) & & 0.663 & & 0.969 & & 0.622 & & $<0.001 \mathrm{a}$ \\
\hline Negative & 38 (46.9) & & $25(30.9)$ & & $30(37.0)$ & & $20(24.7)$ & \\
\hline Positive & $104(49.8)$ & & $65(31.1)$ & & $84(40.2)$ & & $19(9.1)$ & \\
\hline $\begin{array}{l}\text { HER2 overexpression, } \\
\mathrm{n}(\%)\end{array}$ & & 0.954 & & 0.323 & & 0.216 & & 0.101 \\
\hline Negative & $56(47.9)$ & & $32(27.4)$ & & $42(35.9)$ & & $11(9.4)$ & \\
\hline Positive & $55(48.2)$ & & $38(33.3)$ & & $50(43.9)$ & & $19(16.7)$ & \\
\hline $\mathrm{Ki}-67, \mathrm{n}(\%)$ & & 0.182 & & 0.334 & & 0.992 & & 0.503 \\
\hline$<14 \%$ & $93(46.5)$ & & $59(29.5)$ & & $79(39.5)$ & & $25(12.5)$ & \\
\hline$\geq 14 \%$ & $50(54.9)$ & & $32(35.2)$ & & $36(39.6)$ & & $14(15.4)$ & \\
\hline Molecular subtype, n (\%) & & 0.680 & & 0.467 & & 0.671 & & $<0.001^{\mathrm{a}}$ \\
\hline Luminal A & $56(49.6)$ & & $30(26.5)$ & & $44(38.9)$ & & $13(11.5)$ & \\
\hline Luminal B & $56(50.9)$ & & $38(34.5)$ & & $46(41.8)$ & & $8(7.3)$ & \\
\hline HER2 & $10(38.5)$ & & $10(38.5)$ & & $11(42.3)$ & & $11(42.3)$ & \\
\hline Basal-like & $19(52.8)$ & & $10(27.8)$ & & $11(30.6)$ & & $7(19.4)$ & \\
\hline IT, n (\%) & & $<0.001^{\mathrm{a}}$ & & $0.021 \mathrm{a}$ & & $0.004^{\mathrm{a}}$ & & 0.199 \\
\hline Negative & $11(21.5)$ & & $9(17.6)$ & & $11(21.6)$ & & $4(7.8)$ & \\
\hline Positive & $132(55.0)$ & & $82(34.2)$ & & $104(43.3)$ & & $35(14.6)$ & \\
\hline $\mathrm{PT}, \mathrm{n}(\%)$ & & $<0.001^{\mathrm{a}}$ & & 0.130 & & $0.041^{\mathrm{a}}$ & & 0.229 \\
\hline Negative & $6(19.4)$ & & $6(19.4)$ & & $7(22.6)$ & & $2(6.5)$ & \\
\hline Positive & $137(52.7)$ & & $85(32.7)$ & & $108(41.5)$ & & $37(14.2)$ & \\
\hline
\end{tabular}

${ }^{a}$ Denotes statistical significance $(\mathrm{P}<0.05)$. LN, lymph node; LVI, lymphovascular invasion; ER, estrogen receptor; PR, progesterone receptor; HER2, human epidermal growth factor receptor 2; IT, intratumoral inflammation; PT, peritumoral inflammation; LOX, lysyl oxidase; LOXL, LOX-like protein. 


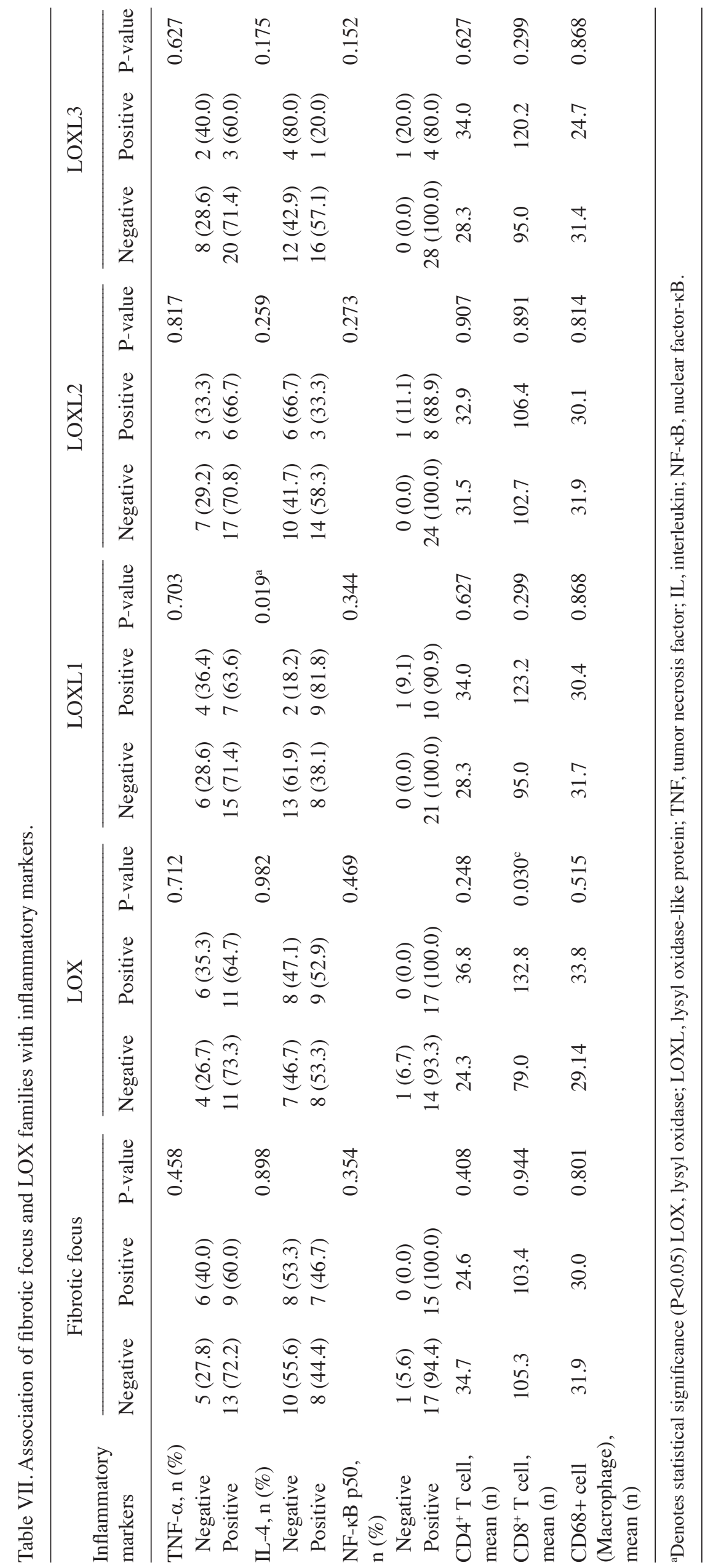



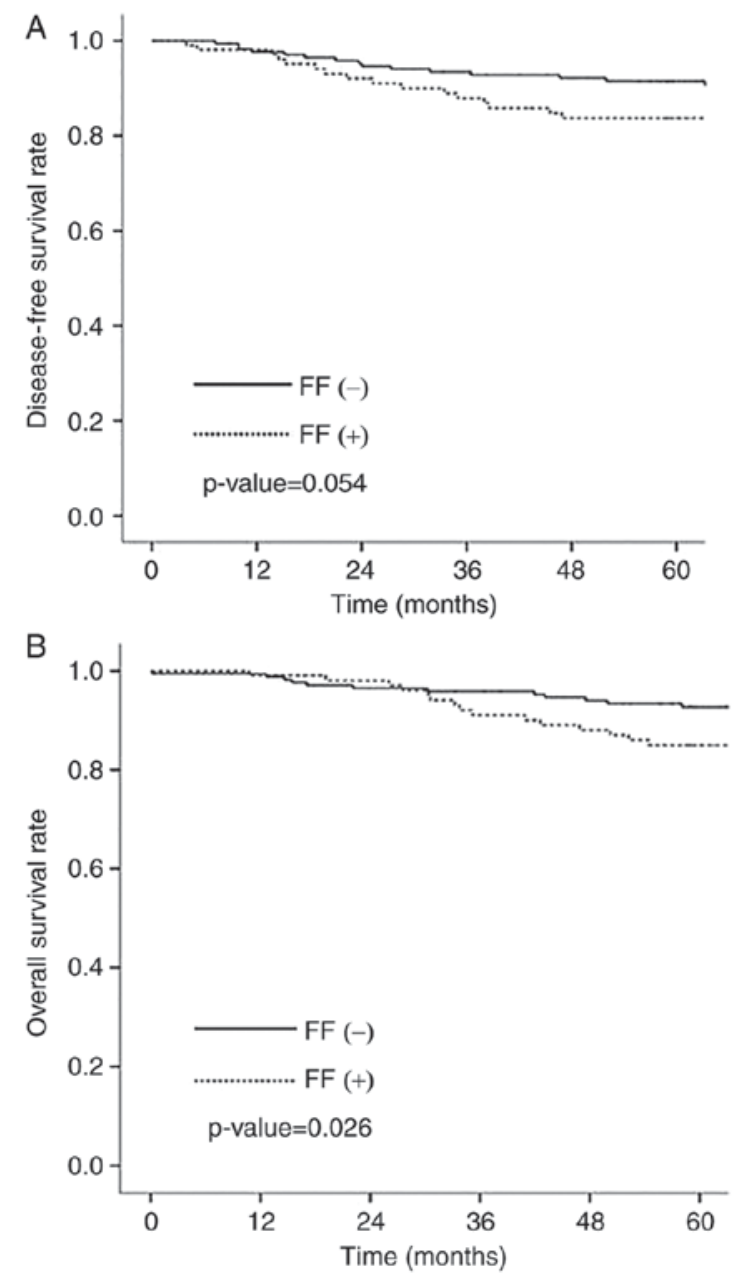

Figure 3. Association of fibrotic focus with patient outcome. (A) disease-free survival, and (B) overall survival.

and T-lymphocytes in fibrotic tissue $(18,25,26)$. Also, various inflammatory mediators including TGF- $\beta$, TNF- $\alpha$, IL-4 and $\mathrm{NF}-\kappa \mathrm{B}$ have been demonstrated to regulate the inflammatory responses as well as the fibrotic signaling cascade $(18,19)$. TNF- $\alpha$ is crucial for the induction of NF- $\mathrm{B}$ (27), the major factor in the inflammatory response and activated NF- $\mathrm{NB}$ is known to contribute to organ fibrosis and cancer development $(19,28,29)$. Furthermore, hypoxia in fibrosis and tumor microenvironment cooperates with inflammatory response in the induction of EMT process $(19,30,31)$. Interestingly, hypoxia induces LOX expression in tumor cells (32) and contributes to promote fibrogenesis and tumor progression through the reinforcement of the EMT process $(19,33)$. In this study, we analyzed the association between FF, the members of the LOX family and inflammatory response. Although the results did not show any association between FF and inflammatory markers, there was significant correlation between $\mathrm{LOX}$ and $\mathrm{CD} 8^{+} \mathrm{T}$ cell. $\mathrm{CD}^{+} \mathrm{T}$ cells are important for the adaptive immune responses and have antitumor effects through various mechanisms. One of the mechanisms, $\mathrm{CD}^{+} \mathrm{T}$ cells secret cytokines such as TNF- $\alpha$ and IFN- $\gamma$, and LOX is known to be up-regulated by cytokines including TNF- $\alpha$ (23). In this regards, $\mathrm{CD}^{+} \mathrm{T}$ cells may affect the expression of LOX. Also, our results showed significant association between LOXL1 and IL-4. IL-4 is a T-lymphocyte-associated cytokine that is involved in humoral and adaptive immunity (34). IL-4 has many biological effects and affects various cell types (34). Interestingly, IL-4 stimulates fibroblasts proliferation 34 and LOXL1 is secreted by fibrogenic cells including fibroblasts (9). Although the mechanism of regulation of LOXL1 has not been fully elucidated, our results suggest that IL-4 may affect the expression of LOXL1.

In previous studies, FF has been reported to be associated with poor prognosis in breast cancer (17). Hasebe et al (35) have demonstrated that the presence of a FF was associated with larger tumor size, high histologic grade, lymph and blood vessel invasion, presence of lymph node metastases, pTNM stage and a shorter DFS and OS in survival analysis. In consistent with the results of previous studies, our results showed that the presence of a FF is associated with more aggressive characteristics of breast cancer. FF was statistically significantly associated with tumor stage, larger tumor size, lymph node metastasis, high histologic grade and p53. Also, OS and DFS were statistically significantly longer in negative FF group than in positive FF group in this study.

In recent years, diverse functions of LOX have been revealed, and LOX has emerged as therapeutic target in cancer therapy. Many pre-clinical studies have described that LOX promotes tumor progression and metastasis $(5,13,36,37)$. In clinical studies, most of studies have also shown that LOX and LOXL expression correlated with a poor prognosis in cancer $(13,14,38-40)$, although study methods were somewhat different from each other. In our study, however, LOX as well as LOXL1 and LOXL2 did not show clinical relevance except LOXL3. Nevertheless, our study describes for the first time that LOXL3 was significantly associated with positive expression of ER and PR and molecular subtype in breast cancer.

Limitations of the present study include the use of the paraffin-embedded tumor tissues in detecting FF and the members of the LOX family, but the fresh frozen tissue in analyzing inflammatory mediators. Tissue processing can cause genetic alterations and molecular changes in tissues (41), and the levels of mRNA transcripts or protein in the paraffin-embedded tissues can be different from those in fresh frozen tissue. Also, we employed immunohistochemical staining to assess the levels of the members of the LOX family, but this method is semiquantitative and less accurate than other objective methods such as quantitative fluorescence analysis or real-time quantitative PCR. Furthermore, we did not include control group such as normal or benign breast tissue. A comparative study between tumor tissue and control group may be necessary to clarify the role of LOX and FF in breast cancer.

In conclusion, our study showed that FF and LOX family are associated with inflammation in breast cancer, although there is no direct correlation between FF and the members of the LOX family. In consistent with the previous studies, our results showed that FF is associated with poor prognosis. And for the first time, we found that LOXL3 was significantly associated with positive expression of ER and PR and molecular subtype in breast cancer. Further studies are required to clarify the mechanisms relating to the LOX family, FF and inflammation in breast cancer.

\section{Acknowledgements}

This study was supported by the grant of Research Institute of Medical Science, Catholic University of Daegu (2013). 


\section{References}

1. Pupa SM, Ménard S, Forti S and Tagliabue E: New insights into the role of extracellular matrix during tumor onset and progression. J Cell Physiol 192: 259-267, 2002.

2. Levental KR, Yu H, Kass L, Lakins JN, Egeblad M, Erler JT, Fong SFT, Csiszar K, Giaccia A, Weninger W, et al: Matrix crosslinking forces tumor progression by enhancing integrin signaling. Cell 139: 891-906, 2009.

3. Gilkes DM, Semenza GL and Wirtz D: Hypoxia and the extracellular matrix: Drivers of tumour metastasis. Nat Rev Cancer 14 430-439, 2014.

4. Acerbi I, Cassereau L, Dean I, Shi Q, Au A, Park C, Chen YY, Liphardt J, Hwang ES and Weaver VM: Human breast cancer invasion and aggression correlates with ECM stiffening and immune cell infiltration. Integr Biol (Camb) 7: 1120-1134, 2015.

5. Cox TR, Bird D, Baker AM, Barker HE, Ho MW, Lang G and Erler JT: LOX-mediated collagen crosslinking is responsible for fibrosis-enhanced metastasis. Cancer Res 73: 1721-1732, 2013.

6. Csiszar K: Lysyl oxidase: A novel multifunctional amine oxidase family. Prog Nucleic Acid Res Mol Biol 70: 1-32, 2001.

7. Payne SL, Hendrix MJC and Kirschmann DA: Paradoxical roles for lysyl oxidases in cancer-a prospect. J Cell Biochem 101: 1338-1354, 2007.

8. Smith-Mungo LI and Kagan HM: Lysyl oxidase: Properties, regulation and multiple functions in biology. Matrix Biol 16 387-398, 1998.

9. Kagan HM and Li W: Lysyl oxidase: Properties, specificity, and biological roles inside and outside of the cell. J Cell Biochem 88: 660-672, 2003.

10. Molnar J, Fong KSK, He QP, Hayashi K, Kim Y, Fong SF, Fogelgren B, Szauter KM, Mink M and Csiszar K: Structural and functional diversity of lysyl oxidase and the LOX-like proteins. Biochim Biophys Acta 1647: 220-224, 2003.

11. Lucero HA and Kagan HM: Lysyl oxidase: An oxidative enzyme and effector of cell function. Cell Mol Life Sci 63: 2304-2316, 2006.

12. Baker AM, Cox TR, Bird D, Lang G, Murray GI, Sun XF, Southall SM, Wilson JR and Erler JT: The role of lysyl oxidase in SRC-dependent proliferation and metastasis of colorectal cancer. J Natl Cancer Inst 103: 407-424, 2011

13. Erler JT, Bennewith KL, Nicolau M, Dornhöfer N, Kong C, Le QT, Chi JT, Jeffrey SS and Giaccia AJ: Lysyl oxidase is essential for hypoxia-induced metastasis. Nature 440: 1222-1226, 2006.

14. Barker HE, Chang J, Cox TR, Lang G, Bird D, Nicolau M, Evans HR, Gartland A and Erler JT: LOXL2-mediated matrix remodeling in metastasis and mammary gland involution. Cancer Res 71: 1561-1572, 2011.

15. Cox TR and Erler JT: Remodeling and homeostasis of the extracellular matrix: Implications for fibrotic diseases and cancer. Dis Model Mech 4: 165-178, 2011

16. Hasebe T, Tsuda H, Hirohashi S, Shimosato Y, Iwai M, Imoto S and Mukai K: Fibrotic focus in invasive ductal carcinoma: An indicator of high tumor aggressiveness. Jpn J Cancer Res 87: 385-394, 1996

17. Van den Eynden GG, Colpaert CG, Couvelard A, Pezzella F, Dirix LY, Vermeulen PB, Van Marck EA and Hasbe T: A fibrotic focus is a prognostic factor and a surrogate marker for hypoxia and (lymph)angiogenesis in breast cancer: Review of the literature and proposal on the criteria of evaluation. Histopathology 51 : 440-451, 2007

18. Ueha S, Shand FH and Matsushima K: Cellular and molecular mechanisms of chronic inflammation-associated organ fibrosis. Front Immunol 3: 71, 2012.

19. López-Novoa JM and Nieto MA: Inflammation and EMT: An alliance towards organ fibrosis and cancer progression. EMBO Mol Med 1: 303-314, 2009.

20. Jeong YJ, Bong JG, Park SH, Choi JI and Oh HK: Expression of leptin, leptin receptor, adiponectin, and adiponectin receptor in ductal carcinoma in situ and invasive breast cancer. J Breast Cancer 14: 96-103, 2011.

21. Radisky DC, Kenny PA and Bissell MJ: Fibrosis and cancer: Do myofibroblasts come also from epithelial cells via EMT? J Cell Biochem 101: 830-839, 2007.

22. Ertz N: Cancer: Opening LOX to metastasis. Nature 522: 41-42, 2015.
23. Nishioka T, Eustace A and West C: Lysyl oxidase: From basic science to future cancer treatment. Cell Struct Funct 37: 75-80, 2012.

24. Leight JL, Wozniak MA, Chen S, Lynch ML and Chen CS: Matrix rigidity regulates a switch between TGF- $\beta$ 1-induced apoptosis and epithelial-mesenchymal transition. Mol Biol Cell 23: 781-791, 2012.

25. Wynn TA and Barron L: Macrophages: Master regulators of inflammation and fibrosis. Semin Liver Dis 30: 245-257, 2010.

26. Luzina IG, Todd NW, Iacono AT and Atamas SP: Roles of T lymphocytes in pulmonary fibrosis. J Leuko Biol 83: 237-244, 2008.

27. Meldrum KK, Metcalfe P, Leslie JA, Misseri R, Hile KL and Meldrum DR: TNF-alpha neutralization decreases nuclear factor-kappaB activation and apoptosis during renal obstruction. J Surg Res 131: 181-188, 2006.

28. Tashiro K, Tamada S, Kuwabara N, Komiya T, Takekida K, Asai T, Iwao H, Sugimura K, Matsumura Y, Takaoka M, et al: Attenuation of renal fibrosis by proteasome inhibition in rat obstructive nephropathy: Possible role of nuclear factor kappaB. Int J Mol Med 12: 587-592, 2003.

29. Karin M and Greten FR: NF-kappaB. Linking inflammation and immunity to cancer development and progression. Nat Rev Immunol 5: 749-759, 2005.

30. Julien S, Puig I, Caretti E, Bonaventure J, Nelles L, van Roy F, Dargemont C, de Herreros AG, Bellacosa A and Larue L: Activation of NF-kappaB by Akt upregulates Snail expression and induces epithelium mesenchyme transition. Oncogene 26: $7445-7456,2007$

31. Kim HJ, Litzenburger BC, Cui X, Delgado DA, Grabiner BC, Lin X, Lewis MT, Gottardis MM, Wong TW, Attar RM, et al: Constitutively active type I insulin-like growth factor receptor causes transformation and xenograft growth of immortalized mammary epithelial cells and is accompanied by an epithelial-to-mesenchymal transition mediated by NF-kappaB and snail. Mol Cell Biol 27: 3165-3175, 2007.

32. Denko NC, Fontana LA, Hudson KM, Sutphin PD, Raychaudhuri S, Altman R and Giaccia AJ: Investigating hypoxic tumor physiology through gene expression patterns. Oncogene 22: 5907-5914, 2003.

33. Higgins DF, Kimura K, Bernhardt WM, Shrimanker N, Akai Y, Hohenstein B, Saito Y, Johnson RS, Kretzler M, Cohen CD, et al: Hypoxia promotes fibrogenesis in vivo via HIF-1 stimulation of epithelial-to-mesenchymal transition. J Clin Invest 117: 3810-3820, 2007

34. Nagai S and Toi M: Interleukin-4 and breast cancer. Breast Cancer 7: 181-186, 2000.

35. Hasebe T, Sasaki S, Imoto S, Muki K, Yokose T and Ochiai A Prognostic significance of fibrotic focus in invasive ductal carcinoma of the breast: A prospective observational study. Mod Pathol 15: 502-516, 2002.

36. Hollosi P, Yakushiji JK, Fong KS, Csiszar K and Fong SF: Lysyl oxidase-like 2 promotes migration in noninvasive breast cancer cells no in normal breast epithelial cells. Int J Cancer 125: 318-327, 2009.

37. Kirschmann DA, Seftor EA, Fong SF, Nieva DR, Sullivan CM, Edwards EM, Sommer P, Csiszar K and Hendrix MJ: A molecular role for lysyl oxidase in breast cancer invasion. Cancer Res 62: 4478-4483, 2002.

38. Hellman J, Jansen MP, Ruigrok-Ritstier K, van Staveren IL, Look MP, Meijer-van Gelder ME, Sieuwerts AM, Klijn JG, SleiJfer S, Foekens JA and Berns EM: Association of an extracellular matrix gene cluster with breast cancer prognosis and endocrine therapy response. Clin Cancer Res 14: 5555-5564, 2008.

39. Patani N, Jiang W, Newbold R and Mokbel K: Prognostic implications of carboxyl-terminus of Hsc70 interacting protein and lysyl-oxidase expression in human breast cancer. J Carcinog 9: 9,2010.

40. Peinado H, Moreno-Bueno G, Hardisson D, Pérez-Gómez E, Santos V, Mendiola M, de Diego JI, Nistal M, Quintanilla M, Portillo F and Cano A: Lysyl oxidase-like 2 as a new poor prognosis marker of squamous cell carcinomas. Cancer Res 68 : 4541-4550, 2008

41. Mc Sherry EA, Mc Goldrick A, Kay EW, Hopkins AM, Gallagher WM and Dervan PA: Formalin-fixed paraffin-embedded clinical tissues show spurious copy number changes in array-CGH profiles. Clin Genet 72: 441-447, 2007. 